\title{
Hacia nuevas formas de pensar el territorio: una propuesta para el desarrollo de la Región del Gran La Plata, Buenos Aires, Argentina
}

\author{
Birche, Mariana \\ Becaria posdoctoral CONICET y docente en \\ la FAU-UNLP y en la FI-UNLP. Instituto de \\ Investigaciones y Políticas del Ambiente \\ Construido (IIPAC) - CONICET/UNLP. ma- \\ rianabirche@gmail.com \\ Rodríguez Tarducci, Rocío \\ Becaria Posdoctoral CONICET y docente en \\ la Universidad Nacional Arturo Jauretche. \\ Instituto de Investigaciones y Políticas del \\ Ambiente Construido (IIPAC) - CONICET/ \\ UNLP. rociotarducci@gmail.com

\section{Jensen, Karina} \\ Becaria Posdoctoral CONICET y docente en \\ la cátedra de Teorías y Planificación Terri- \\ torial Meda-Altamirano-Yantorno. Instituto \\ de Investigaciones y Políticas del Ambiente \\ Construido (IIPAC) - CONICET/UNLP. kari- \\ nacjensen@gmail.com
}

\section{Cremaschi, María Elisa}

Becaria Doctoral CONICET y docente investigadora categoría $\mathrm{V}$ en la FAU-UNLP (cátedra de Procesos Constructivos Cremaschi-Sáenz). Instituto de Investigaciones y Políticas del Ambiente Construido (IIPAC) CONICET/UNLP. elisacre@hotmail.com

\section{Bilbao, Pilar}

Estudiante del último año en la carrera Arquitectura y Urbanismo. Beca de Entrenamiento de Investigación FAU. Ayudante alumno en la FAU-UNLP (cátedra de Historia de la Arquitectura 3 Gandolfi-Aliata-Gentile). Instituto de Investigaciones y Políticas del Ambiente Construido (IIPAC) CONICET/UNLP. pilibilbao@gmail.com

\section{Ravella, Olga}

Profesora consulta en la Facultad de Arquitectura y Urbanismo de la Universidad Nacional de La Plata. Instituto de Investigac iones y Políticas del Ambiente Construido (IIPAC) - CONICET/UNLP. olga.ravella@ gmail.com 
Hacia nuevas formas de pensar el territorio: una propuesta para el desarrollo de la Región del Gran La Plata, Buenos Aires, Argentina

\section{Resumen}

El presente artículo se ocupa de analizar el desarrollo de la Región de La Plata desde la fundación a la actualidad, para luego proponer propuestas de alternativas tendientes a mitigar los efectos provocados por el desarrollo urbano insustentable. Para esto, se discuten los principios considerados como "sustentables" y se indaga sobre cómo su aplicación puede constituirse en una estrategia de abordaje del desarrollo sustentable, en el contexto del actual paradigma técnico-económico y socio-ambiental. Como resultado de la investigación se conciben propuestas alternativas de desarrollo a partir de las diversas investigaciones realizadas por los autores en los últimos veinte años.

Palabras clave

Sustentabilidad urbana; desarrollo territorial; planificación territorial.

\section{Towards new ways of thinking about territory: a proposal for the development of the Gran La Plata region, Buenos Aires, Argentina}

\begin{abstract}
This article deals with analyzing the development of the La Plata Region from its foundation to the present, to propose alternative aimed at mitigating the effects caused by unsustainable urban development. For this, the principles considered as "sustainable" are discussed and it is investigated how their application can become a strategy for approaching sustainable development, in the context of the current technical-economic and socio-environmental paradigm.

As a result of the research, alternative development proposals are conceived from the various investigations carried out by the authors in the last twenty years.
\end{abstract}

\section{Keywords}

Urban sustainability; territorial development; territorial planning.

\section{Rumo a novas formas de pensar o território: Uma proposta para o desenvolvimento da região do Gran La Plata, Buenos Aires, Argentina}

\section{Resumo}

Este artigo trata de analisar o desenvolvimento da Região de La Plata desde a sua fundação até o presente, para então propor alternativas destinadas a mitigar os efeitos causados pelo desenvolvimento urbano insustentável. Para tanto, são discutidos os princípios considerados "sustentáveis" e investiga se como a aplicação desses princípios pode se tornar uma estratégia para a abordagem do desenvolvimento sustentável, no contexto do atual paradigma técnico-econômico e socioambiental. Como resultado da pesquisa, propostas de desenvolvimento alternativo são concebidas a partir das várias investigações realizadas pelos autores nos últimos vinte anos.

\section{Palavras-chave}

Sustentabilidade urbana; desenvolvimento territorial; planejamento territorial 


\section{Introducción}

En la actualidad se observa que toda intervención en el territorio se considera desde la perspectiva de la "sustentabilidad-sostenibilidad". Este término de tanta vaguedad e imprecisión se incluye en las propuestas de formulación de estrategias y políticas generales para alcanzar ciudades “inclusivas, seguras, resilientes y sostenibles” antes de $2030^{1}$, o para denominar distintas acciones parciales desde el urbanismo hasta la vivienda, la movilidad, el transporte público o el paisaje. Esto supone el desconocimiento acerca de que dichos criterios fueron utilizados para pensar las ciudades desde mediados del siglo XIX. Asimismo, es usual que no se precise ni profundice en cómo y en cuánto dichas intervenciones podrían aportar a mejorar la calidad físico-espacial y socioeconómica de las ciudades, colaborando en disminuir la insustentabilidad integral. A partir de estas reflexiones se analizó el proceso de producción del espacio urbano-territorial de la Región del Gran La Plata, constituida por tres ciudades: La Plata, Berisso y Ensenada. El diseño de la nueva capital provincial fue desarrollado ex novo, y contenía un conjunto de criterios que actualmente se consideran "sustentables", y que en el transcurso del tiempo fueron incorporando actividades y formas de crecimiento que generan impactos negativos tanto ambientales como económicos y socio-espaciales.

En este artículo se presentan algunos criterios pensados desde las perspectivas económicas, ambientales, sociales y físico-espaciales, para la formulación de alternativas de planificación urbano-territorial y de diseño de nuevos espacios para el ensanche físico-urbanístico de la Región del Gran La Plata. Estas alternativas buscan posibilitar un mejoramiento en la calidad de vida de los habitantes en general y de los sectores más desfavorecidos en particular, así como también tender a una organización espacial menos desequilibrada de la región en estudio.

Los criterios incluyen variables contempladas en el diseño original de la ciudad y otros que provienen de los lineamientos planteados por la Agenda 2030. En este sentido, desde la visión urbanística se plantea la siguiente pregunta: ¿cuáles serían los modelos de organización y crecimiento urbano que respondan a los requerimientos del modelo económico social y ambiental actual, que mejoren la calidad de vida urbana? Para intentar dar respuesta a esta pregunta, la metodología utilizada se basó en la combinación de dos estrategias de tipo cualitativas. En primer lugar, el análisis de contenido de un corpus documental conformado por los propios autores, que con distintas metodologías produjeron resultados
1. Objetivo de Desarrollo Sostenible $N .^{\circ} 11$ de la Nueva Agenda Urbana Asamblea General de las Naciones Unidas en el marco de los Objetivos de Desarrollo Sostenible período 2016-2030. 
acerca de la interacción entre políticas públicas, desarrollo urbano, déficits habitacionales, la problemática territorial desde el ambiente, la pobreza y la salud, entre otros. En segundo lugar, el análisis de un conjunto de material bibliográfico e investigaciones relativas a: i. los emergentes productivos, sociales y económicos que afectan al trabajo y a la calidad de vida de las personas; ii. los desarrollos teóricos y el análisis de problemáticas socio-espaciales más relevantes y iii. los problemas derivados del cambio climático, desde un enfoque interpretativo (CÁ́ceres, 2003).

En cuanto a la organización del presente artículo y para una mejor comprensión, consta de cuatro apartados: el primero busca interpretar algunas cuestiones teóricas relacionadas con conceptos como sustentabilidad/insustentabilidad, desarrollo y emergentes económicos y sociales. El segundo pone de manifiesto los procesos de insustentabilidad producidos en la Región del Gran La Plata desde su creación y al mismo tiempo determina aquellas fortalezas y debilidades presentes en el territorio. En el tercer apartado se presentan los criterios y propuestas de intervención, producto del análisis histórico y el diagnóstico realizados durante el segundo apartado. Se retoman los principios teóricos acerca de la sustentabilidad presentados en el primer apartado, reflejados también en el segundo, durante la revisión de la etapa fundacional de ciudad de La Plata. El cuarto y último apartado reflexiona a modo de cierre sobre el recorrido realizado durante los primeros tres, y desarrolla una discusión acerca del modo más adecuado para intervenir en las ciudades, planteando alternativas de planificación territorial con el objetivo de mejorar las condiciones de vida de la población y mitigar problemáticas a futuro.

\section{La ecología y la sustentabilidad en el desarrollo urbano}

Desde el inicio de la historia, las acciones de los grupos humanos produjeron impactos en la naturaleza, pero a partir de la Revolución Industrial el modelo productivo capitalista se basó en procesos altamente dependientes de las energías no renovables y de la depredación de los recursos naturales. Esta situación fue considerada por un conjunto de economistas denominados fisiócratas, entre los cuales François Quesnay (1694-1774) pretendía que era necesario acrecentar la producción de "riquezas renacientes" (o renovables) sin deteriorar los "bienes de fondo", lo que dio lugar a su noción de producto neto o renta (NAREDo, 1996). Desde entonces la civilización industrial optó por el desarrollo energo-productivo intensivo utilizando el capital material como parte del capital natural a través de la simple explotación y deterioro 
de ese potencial, lo que provocó graves consecuencias ecológicas y ambientales. A partir de entonces, la ciencia económica se construyó adoptando como propio ese modelo de desarrollo y abandonando el objetivo de aquellos primeros economistas. El impacto negativo del modelo energo-intensivo sobre los ecosistemas comenzó a ser tratado desde mediados del siglo XIX, planteando propuestas para mejorar la calidad de vida de las personas y del funcionamiento de las ciudades. Aquellas concepciones finalmente se institucionalizaron en la Conferencia de Estocolmo de 1972 y el Informe del Club de Roma ${ }^{1}$, y sugirieron la necesidad de modificar determinadas acciones para disminuir los impactos provocados por el cambio climático.

Desde fines del siglo XIX, la ecología aportó conocimientos relacionados con el comportamiento de los sistemas naturales, cuyos presupuestos se comenzaron a utilizar para analizar el desarrollo urbano. Desde esa disciplina Ignacy $\mathrm{SACHS}^{2}$ promovía utilizar los conceptos del ecodesarrollo para aplicar a los procesos del crecimiento socio-económico. Este concepto en la década 1980 fue suplantado por "desarrollo sustentable", término que proviene

del empeño de conciliar crecimiento (o desarrollo) económico con la idea de sustentabilidad, cuando cada uno de estos conceptos se refiere a niveles de abstracción y sistemas de razonamiento diferentes. La idea de crecimiento o desarrollo económico se encuentra hoy desvinculada del mundo físico, y no tiene otro significado concreto y susceptible de medirse que el referido al aumento de los agregados de Renta o Producto Nacional (NAREDo, 1996).

Estas definiciones han sido discutidas por su ambigüedad, y fueron redefinidas desde las diferentes disciplinas. Entre ellas se incluyeron conceptos como igualdad, equidad e inclusión, conjuntamente con otros aspectos físicos y espaciales del funcionamiento de las ciudades. A efectos de analizar si los principios planteados como parte del desarrollo sustentable se cumplen cuando alguno de ellos se aplica a los planes territoriales y urbanísticos se consideran las siguientes premisas básicas:

1. En todo proceso productivo no solo debe considerarse el capital monetario, sino fundamentalmente el capital natural (recursos naturales de la tierra), para asegurar que el valor de la inversión en el stock de capital productivo cubra, al menos, la valoración anual de su deterioro. En este sentido, la idea de que ciertas mejoras puedan lograrse a escala local o regional no invalida el presupuesto de la incapacidad del sistema de arreglar internamente el deterioro causado por su propio funcionamiento y de llevar estas mejoras a nivel global, teniendo en cuenta que se importa materia prima y energía de los mismos territorios a los que se les envían residuos y materias contaminantes (NAREDo, 1996). 
2. En los sistemas urbanos en los que fundamentalmente se desarrolla la vida y la economía de los seres humanos, disminuir la insustentabilidad se lograría, según CaLvo y SANCHO (2001, en HenRíquez 2004), a través de un cambio radical de actitud de la sociedad y del paradigma económico, hacia un modelo centrado en lo ecológico y en la calidad de vida. Esta última aproximación incorpora la dimensión social, ya que solo estructuras sociales estables, democráticas, solidarias intra e intergeneracionales, libres y justas serán capaces de ser sostenibles. A esta visión se la denomina sustentabilidad fuerte y se diferencia de la sustentabilidad débil (PeArce y Atkinson, 1993, en Henríquez, 2004). Refiriéndose a los sistemas físicos sobre los que se organiza la vida de las personas (agrarios, industriales, urbanos) NAREDO (1996) afirma que la sustentabilidad de tales sistemas dependerá de la posibilidad que tienen de abastecerse de recursos y de deshacerse de residuos, asî como la posibilidad de controlar las pérdidas de calidad (tanto interna como ambiental, que afectan su funcionamiento). La conservación de determinados elementos o de sistemas integrantes del patrimonio natural no solo necesita ser asumida por la población, sino que requiere instituciones que velen por la conservación y transmisión de ese patrimonio a las generaciones futuras.

3. Desde otra óptica, el actual paradigma técnico-económico basado en la informática y las nuevas tecnologías provoca otras situaciones que agravan el proceso de insustentabilidad y se relacionan con los criterios de igualdad, inclusión, equidad, planteados como bases para la sustentabilidad. Se trata de la pérdida de puestos de trabajo a medida que se incluyen las nuevas tecnologías de la información y la robótica a las actividades productivas y de servicios, lo que provoca la desocupación de miles de personas. En este contexto pensar en una trayectoria "sustentable" resulta difícil.

En estas condiciones, para lograr disminuir la insustentabilidad y conseguir un desarrollo más equilibrado, se requeriría aplicar un conjunto de principios y acciones que solo serán posibles de lograr con una modificación estructural del sistema económico y de la decisión del sistema político.

\section{El proceso de desarrollo en el Gran La Plata}

La denominada Región del Gran La Plata (figura 1) corresponde al conglomerado urbano que forma parte Área Metropolitana de Buenos Aires (AMBA) e incluye los partidos de Ensenada, Berisso y La Plata (capital de la provincia de Buenos Aires). Entre los tres partidos suman 900.000 habitantes, aproximadamente, de acuerdo con proyecciones a partir 
de los datos oficiales del último censo del año 2010. Entre las principales características de la región se encuentra su estructura socioeconómica definida por un significativo peso del sector terciario, en particular, el sector de la administración pública, concentrado fundamentalmente dentro de los límites del casco fundacional de la ciudad planificada de La Plata. Dado el carácter de capital provincial, sede de la Universidad Nacional de La Plata y de numerosas entidades empresariales y financieras, también se caracteriza por la importancia de grandes industrias de las ramas metalmecánica y petrolera emplazadas sobre el área portuaria: la destilería y petroquímica Repsol-YPF, astilleros Río Santiago y siderurgia Siderar, así como también es un polo de investigación y desarrollo en distintas especialidades.

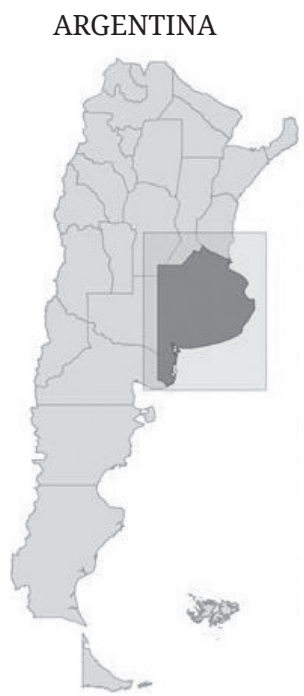

PROVINCIA DE BUENOS AIRES

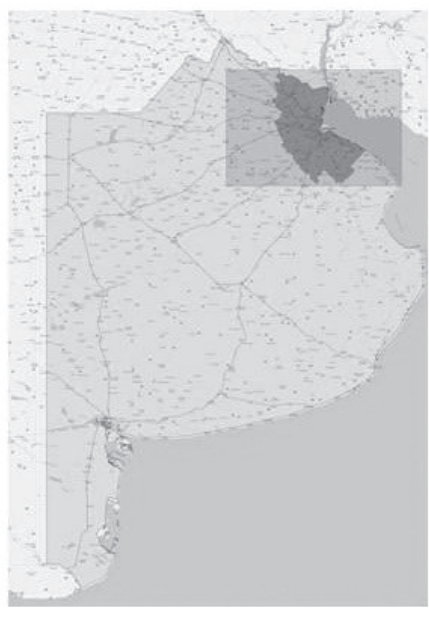

REGIÓN METROPOLITANA

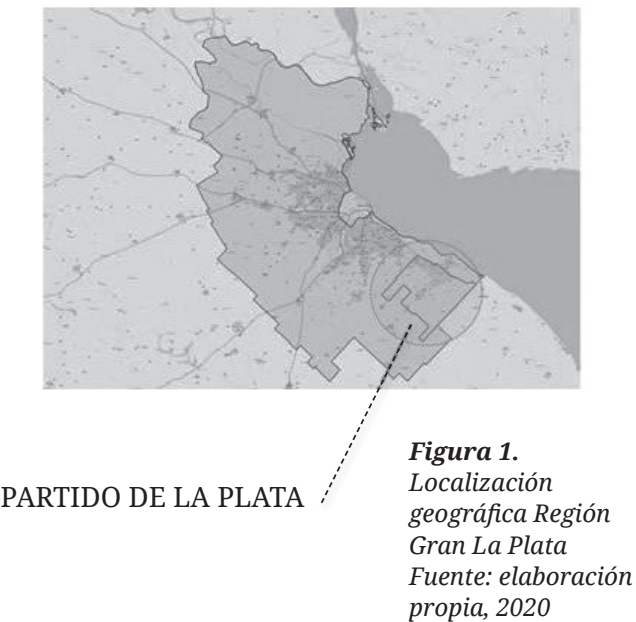

Las características que definen al Gran La Plata como área de estudio tuvieron su inicio en el final del siglo XIX, que significó un tiempo en que la modernidad y la revolución industrial impulsaron el avance de la ingeniería entre dos paradigmas técnico-económicos, el 
basado en el acero barato y el cuarto paradigma, basado en el petróleo barato y la industria automotriz. En este contexto, fue creada la ciudad de La Plata como capital de la provincia de Buenos Aires. Su localización fue producto del conflicto de intereses entre actores que se habían apropiado de un sector del territorio y lo habían dominado, y que requerían la instalación de un puerto.

En consonancia con los presupuestos económicos y sociales de la época, el territorio de la Región del Gran La Plata se constituyó en un ecosistema conformado por una cuenca hídrica, un área para localizar el puerto y un paisaje de gran riqueza arbórea y de diseño, en algunas tierras que eran parte del actual parque Pereyra Iraola. Ya antes de la fundación, el propio gobernador Dardo Rocha advierte la presencia de una cañada o cauce temporal (refiriéndose a uno de los brazos del arroyo El Gato) que podría ocasionar conflictos, y manda trasladar la ciudad unos 1000 metros más al sudeste (VALLEJo, 2005).

La ciudad de La Plata fue pensada con vistas a un gran futuro y construida rápidamente con base en las ideas influenciadas por las corrientes higienistas europeas, principalmente inglesas y las de Frederick Law Olmsted. En este sentido, su fundador, Dardo Rocha, sintetizó el pensamiento de la época resaltando los valores paradigmáticos del siglo XIX:

La ciudad Capital, para que responda a su desenvolvimiento y a su destino, debe ofrecer fácil acceso a los hombres y a los intereses que está llamada a servir, consultar con la higiene que se impone en primer término en nombre de la existencia; las conquistas del arte que llevan el sentimiento de lo bueno y de lo bello; los adelantos de la industria, que agiganta la producción, y los transportes reclamando cada día más amplias avenidas; y las conveniencias del comercio, para que esto concurra a su rápido acrecentamiento (DARDO RochA, 1881).

El centro de interés principal consistió en facilitar el modelo agroexportador a partir de la creación del puerto, facultades de Veterinaria y Agronomía, Museo de Ciencias Naturales para el desarrollo de la investigación en disciplinas anexas y el Colegio Nacional para proveer de jóvenes formados para el ingreso a la universidad.

El trazado de la ciudad se basó en ideas asociadas a las diferentes teorías urbanas del siglo XIX, similares a las actuales "sustentables", tales como la calidad del ecosistema del territorio, la existencia de una de las primeras redes ferroviarias y las vías de comunicación que posibilitaron la vinculación con el interior y el exterior del país. 
El plano fundacional de la ciudad reserva el 58 \% de su superficie para la construcción, el 35 $\%$ para los espacios circulatorios y un $9 \%$ para las plazas y parques, lo que más tarde haría de La Plata la ciudad más verde de Argentina (Gorelik, 2004). Esto implica que los criterios no explicitados en aquel momento histórico corresponden a lo que hoy se podría denominar criterios bioclimáticos, sustentables o eco-urbanísticos.

Además, la ciudad estaba definida por una avenida de circunvalación de cien metros de ancho totalmente arbolada que circunda una malla jerarquizada de vías, calles y avenidas, en cuyas intersecciones se localizan plazas y parques de variadas formas y superficies. Asimismo, se consideró la existencia de corazones de manzana libres, cierta armonía de alturas edilicias, amplias veredas y calles orientadas a medio rumbo con el objetivo de permitir buen asoleamiento de las direcciones norte-sur y este-oeste.

Hacia el nordeste de la vía de circunvalación se marcó la subdivisión de chacras y quintas creando un cinturón frutihortícola destinado al abastecimiento de la ciudad. Fuera del casco central se localizaron el cementerio y los hospitales, considerados para los pensadores del higienizo como incompatibles con el buen funcionamiento del área urbana. Del mismo modo, se previeron en las afueras de la ciudad las localizaciones de hornos de ladrillos, así como algunas actividades industriales, donde se localizó la población de trabajadores de distintas actividades productivas. La ciudad, a pesar de los principios considerados sustentables, surgió fragmentada, los barrios de los sectores de los obreros y la ciudad de la administración, la academia, el arte, tal como considera el postulado de LEFEBvRE que afirmaba que el urbanismo es un fenómeno de clase, el espacio creado por la burguesía es un instrumento que le permite cumplir sus objetivos: dispersar a la clase obrera repartiéndola en los lugares asignados a ella, organizar y subordinar los flujos a las reglas institucionales, en definitiva, controlar el espacio y regir de forma tecnocrática a la sociedad en su conjunto. El concreto espacio de habitar modelado así por el urbanismo es pues irremediablemente político (Lefebvre, 1972, p. 157).

A principios de la década de 1920, la instalación de los frigoríficos en Berisso, los residuos vertidos directamente a las aguas sin tratamiento y la destilería de petróleo YPF en la franja de terreno entre el puerto y la ciudad de La Plata (figura 2) antepusieron el crecimiento económico a las preocupaciones ambientales, y se alejaron de los principios adoptados en la creación de la ciudad, que podría decirse constituía una "sustentabilidad fuerte". Esta decisión se tradujo no solo en el deterioro del propio territorio, sino que afectó también a toda la región. 
Además, la zona denominada paseo del bosque, planteada en sus inicios como un enorme corazón verde, sufre hasta hoy ocupaciones de distintas actividades: loteos, hipódromo, zoológico, edificios universitarios y, por otra parte, el deterioro arbóreo, ya sea por voluntad de la Municipalidad o por enfermedades. Debido a la contaminación ambiental y a la falta de tratamiento, muchos de los árboles de ciudad murieron y continúan muriendo de pie (Ravella \& Giacobbe, 2001). Asimismo, La Plata contó con un cuerpo normativo, pero sus imperfecciones, sus omisiones o, ¿por qué no?, las excepciones acordadas fueron consolidando paulatinamente el actual perfil urbano desarticulado, segmentado, falto de identidad y calidad ambiental.

NUETCIPIO DE LA CAPYTAL, DE BA PROYIKCIA

LA PLATA.
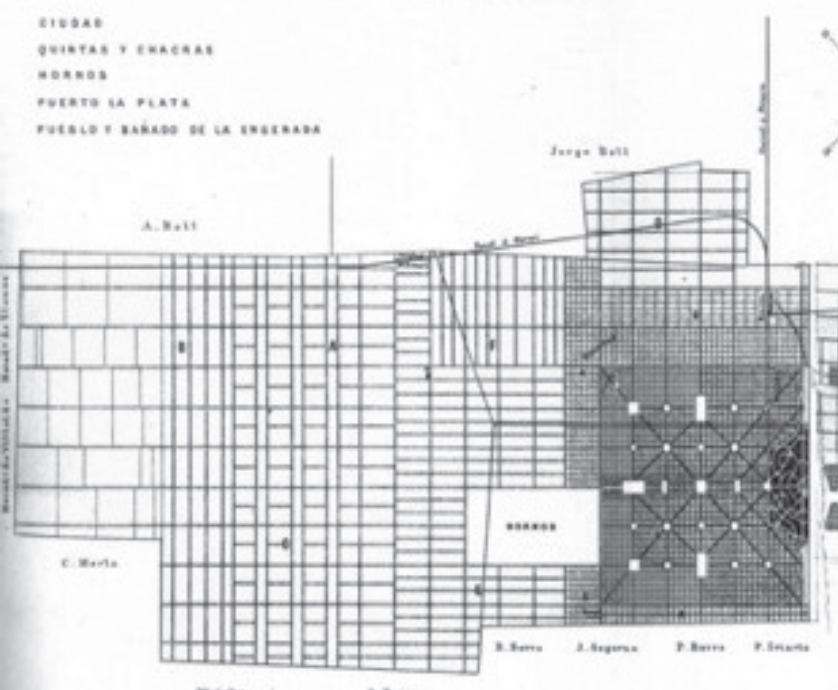

woipes

stomes

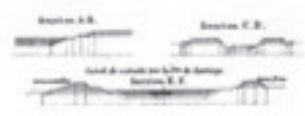

Figura 2. Plano de la fundación de la ciudad de La Plata 1882 Fuente: Archivo General de la Nación, Legajo Nro. 2892 
A partir de la década de 1930, con la consolidación del paradigma técnico-económico basado en el petróleo barato, el medio automotor favoreció la transformación de muchos núcleos urbanos, entre ellos, el crecimiento poblacional hacia las periferias de la ciudad de La Plata. Posteriormente, a mediados de la década de 1950, se consolidó el modelo económico desarrollista que planteaba la necesidad de interrelacionar producción y tecnología. En relación con la tecnología, se crearon organismos de investigación y desarrollo, como el CONICET ${ }^{2}$ y el INTA ${ }^{3}$, que a partir del impulso a la investigación y la relación universidad-producción posibilitaron el crecimiento económico macro a partir de las mejoras técnicas y organizacionales. De este modo se promovió y consolidó la investigación en la Universidad Nacional de La Plata.

Desde la perspectiva urbanística se adoptaron las nuevas concepciones sobre planificación desarrolladas en Europa e impulsadas por la $\mathrm{CEPAL}^{4}$ en América Latina. Estas, conjugadas con el desarrollismo económico dominante en la escena política, crearon el clima necesario para la realización de un plan urbano para la Región del Gran La Plata. De este modo, en 1961, surge el plan diseñado por el grupo URBIS. ${ }^{5}$ En el marco de las dos teorías urbanísticas en pugna en aquel momento, la concentración tipo "ville radieuse" versus ciudad jardín, el plan para La Plata adscribía a la primera, intentando reforzar la función de la capitalidad de La Plata. Sin embargo, la evolución del Gran La Plata no siguió ninguno de estos caminos.

Las poblaciones que se desarrollaron a lo largo del eje que une La Plata con Buenos Aires se conformaron siguiendo las características formales de las ciudades jardín. En el centro del casco fundacional, sin un cuerpo reglamentario coherente que se opusiera a la especulación, se transformó en la norma, se densificó en altura y se avanzó casi monolíticamente hasta el corazón de las manzanas, lo que alteró las tipologías del período fundacional. El resto de la periferia se fue consolidando con un hábitat difuso, de muy baja densidad, carente de identidad. El crecimiento urbano producido en este período para los diferentes sectores socio-profesionales de la población tuvo un denominador común, que fue el loteo privado indiscriminado en detrimento del espacio rural y productivo. Como se puede verificar, las propuestas del plan adscribían a principios que actualmente se constituyen en aquellos que se promueven desde los organismos internacionales para lograr ciudades menos insustentables. Asimismo, se descartaron las ideas urbanísticas del movimiento moderno que dejaron su impronta en la ciudad, con la construcción de algunos conjuntos habitacionales en 1960, cuyos criterios hoy se distinguen como "sustentables".
2. Consejo Nacional de Investigaciones Científicas y Técnicas.

3. Instituto Nacional de Tecnología Agropecuaria.

4. Comisión Económica para América Latina y el Caribe.

5. Plan Urbis (1961). 
A fines de la década de 1960 se evidenció la crisis del Estado de Bienestar, con la emergencia del paradigma técnico-económico basado en la información y la informática, y comenzó globalmente un proceso de desindustrialización que se apoyó en la implementación de políticas neoliberales en economía y de derecha en política. Esta situación y la sustitución del sistema de transporte público tranviario por el automotor posibilitaron el rápido e improvisado crecimiento de las periferias debido a la especulación inmobiliaria, que indujo la producción de tres formas de crecimiento urbano. La primera relacionada con los loteos indiscriminados que realizaron los sectores inmobiliarios en las periferias de la ciudad, a partir de los cuales se asentó la población de medios y bajos recursos que podían acceder a un préstamo hipotecario, sin exigencias de los mínimos servicios de agua, cloacas o pavimento, ni de reservas verdes, espacios para escuelas y otros servicios. Esta situación se verificó a fines del siglo XX con un 23 \% de la población sin conexión a la red de agua corriente y un $37 \%$ sin servicios cloacales (RAvelLa \& GiacobBE, 2001).

La segunda forma de crecimiento se relacionaba con la localización de los sectores de medio-alto y altos recursos, en zonas con o sin servicios, manteniendo reservas para los espacios verdes y públicos, fundamentalmente en las localidades en la franja del corredor vial y ferroviario Buenos Aires-La Plata. La tercera se corresponde con los asentamientos informales, que se ubicaron, salvo excepciones, sobre áreas naturales inadecuadas, que implicaban problemas ambientales y de salubridad para la población. Si bien "sustentabilidad urbana" es un concepto introducido en el discurso oficial internacional desde los años 80 , es en los últimos diez años cuando en Argentina comienza a formar parte del lenguaje común de todas las disciplinas produciendo discursos banalizados. En este contexto, desde las esferas estatales se impulsa la redefinición del rol de la ciudad a partir de importantes realizaciones y proyectos para la región, sin que se visualice un plan de conjunto como parte de una idea global inscripta en básicos principios de sustentabilidad, equilibrio territorial, disminución de la desigualdad socio-espacial, entre otros.

Estas acciones aumentaron los precios del suelo urbano y rural, así como de los alquileres, y expulsaron a sectores medios hacia las periferias. El alza del valor del suelo rural provocó que importantes áreas del suelo productivo fueran decapitadas para ser ofertadas para ese tipo de urbanización. Asimismo, la creación de la autopista Buenos Aires-La Plata, además de valorizar el suelo, provocó su degradación a causa de la extracción profunda de tierra seleccionada, destinada principalmente para rellenos y subrasantes necesarios para su 
ejecución, con la consiguiente aparición de cavas y los riesgos socio-ambientales que trae aparejados (JENSEN, 2018). Estas formas de urbanización aumentaron las variables de insustentabilidad en áreas de alta vulnerabilidad ecológica y ambiental.

A partir de 2001, la gran crisis socio-económica provocada por el neoliberalismo, en Argentina, incrementó los niveles de pobreza al 53 \% y la desocupación al 23 \%, según datos del INDEC. Ya desde 2002, la imposición de un modelo económico basado en la recuperación de las fuentes de trabajo y el control de la importación de bienes, servicios y capitales sin restricciones produjo una recuperación económica que posibilitó el desendeudamiento, el desarrollo exponencial de la ciencia y la tecnología y crecimiento económico post crisis. Este crecimiento se detuvo a partir de 2008, en el contexto de la gran crisis financiera mundial. A pesar de la crisis económica mundial y local, en 2015 la pobreza había disminuido al 27 \% según estudios de la Universidad Católica Argentina. Desde 2008, el país debió adquirir deuda nuevamente; por el mismo motivo se destinó un menor financiamiento a la investigación, la educación y la salud, se aumentó la desocupación del 7,1 \% al 9,7 \% (INDEC) y la pobreza volvió a aumentar al 40 \%. A partir de principios de 2020 y en el escenario de la pandemia, la pobreza aumentó al 44,3 \%, se incrementaron las tomas de tierra y la localización de las urbanizaciones informales en áreas de vulnerabilidad, situación que había aumentado desde $2013^{6}$. En este contexto se constata que alrededor de 200.000 personas (RENABAP, 2018) habitan en aproximadamente 190 urbanizaciones informales entre las distintas tipologías, con graves problemas de saneamiento, falta de servicios urbanos básicos, contaminación ambiental, etc. (RodRíGuez TARDUcci, 2020). Por otra parte, se continúan proyectando propuestas de urbanizaciones cerradas, muchas veces sobre áreas de alta vulnerabilidad ecológica o sobre suelos de alta calidad productiva, como es el caso del eje noroeste del partido de La Plata.

En el caso del casco fundacional de La Plata, se continúa densificando en lotes de 10 x 30 metros en un trazado concebido para densidades medias y cementando los corazones de manzanas, lo que impide la absorción de las aguas de lluvia. Asimismo, las grandes superficies comerciales se localizan sin criterios ambientales, y las redes viarias, tanto las del casco fundacional como las de las periferias, no se adecuaron al crecimiento de la circulación ni a las necesidades del incremento poblacional, lo que provoca parte del caos de tráfico y estacionamiento que hoy es rasgo distintivo de ambos sectores. Sobre la base de los estudios realizados por el equipo de investigación, la Región del Gran La Plata presenta en
6. Esto se constata en el censo INDEC 2010, indicando que mientras el crecimiento de la ciudad de Buenos Aires era del $1,2 \%$, la población de las urbanizaciones informales crece un $3,4 \%$, debido al incremento vegetativo $y$ la inmigración de países vecinos, fundamentalmente. 
la actualidad las siguientes problemáticas económicas, sociales y ambientales que se muestran en la figura 3 (ver página 50).

1. El desarrollo productivo de la región no tuvo en cuenta el impacto sobre el capital natural ni los bienes ecosistémicos. La producción petroquímica provoca graves deterioros de los suelos, contamina el aire y produce residuos tóxicos que afectan a todo el ecosistema del territorio. La producción del área hortícola se realiza en invernaderos y con la utilización de agrotóxicos que, al ocupar grandes extensiones del suelo, impiden la filtración de las aguas de lluvia e influyen en las inundaciones frecuentes de las áreas urbanizadas. Sin embargo, el alto valor productivo de estas tierras se constituye en una fortaleza económica si se promueve la operativización y organización de la producción orgánica. Esta situación puede ser potenciada por la participación de los distintos centros de investigación y desarrollo de la Universidad de La Plata. A pesar de que la situación del desarrollo científico-tecnológico, implica el incremento de los puestos de trabajos propios y derivados de nuevas industrias basadas en el conocimiento y las tecnologías asociadas, y se constituye en un problema para el futuro próximo, ya que el aproximadamente el $50 \%$ de la población de la región no tiene acceso a la educación superior (INDEC, 2020).

2. La situación del ecosistema urbano-natural se ha tornado especialmente crítica. Los efectos del cambio climático aumentaron la vulnerabilidad del ecosistema que, siendo una cuenca hídrica, no ha sido tratada. En consecuencia, resultan más frecuentes e importantes las inundaciones y sequías en períodos más extensos, lo que se traduce en problemas de abastecimiento del agua. Estos temas deben ser considerados, ya que el cambio climático incrementará sus efectos negativos sobre los sistemas urbanos debido al incremento de las temperaturas y sus consecuencias. Entre las consecuencias previstas está la inundación de algunos sectores urbanizados de Berisso y Ensenada, y otras, como la existencia de cavas y la ocupación de áreas de vulnerabilidad ecológica, ya sea por la localización de urbanizaciones cerradas o informales. Del mismo modo, el incremento del turismo local y regional comienza a encontrar en las islas Santiago y Paulino lugares para ser usados, como también el caso de la costa de Punta Lara, que se constituyen en temas que tienen que ser tratados.

3. En cuanto al desarrollo socio-espacial de las distintas localidades de la región, se desvirtuaron los principios primigenios, que actualmente se consideran "sustentables". El crecimiento urbano, tanto del casco fundacional como de las periferias, formales e informales, no fue 
acompañado por el crecimiento y modernización de las redes de agua potable, ni por las redes de desagüe cloacal, lo que incrementa la contaminación de las aguas subterráneas, al mismo tiempo que las urbanizaciones cerradas con la instalación de este tipo de redes particularizadas provocan problemas en sus entornos. Asimismo, no se ampliaron ni modernizaron las redes de electricidad y de comunicación, lo que implica una forma más de inequidad social, por una parte, y por el funcionamiento ineficiente, por otra. El crecimiento y densificación del casco fundacional se producen desvirtuando los principios fundacionales y de "sustentabilidad", acentuado con la inclusión del cementerio considerado como actividad insustentable o las grandes superficies comerciales cementadas, que deterioran asimismo el paisaje urbano. El parque edilicio, asimismo, se produce sin aplicación de las "tecnologías apropiadas" de lo que se denomina arquitectura bioclimática. Ambas situaciones contribuyen a incrementar la insustentabilidad. Por otra parte, dicho crecimiento poblacional determinó la consolidación de nuevas centralidades en las principales localidades, que pueden beneficiar el desarrollo futuro, por posibilitar un mejoramiento de las diversas movilidades.

4. El sistema de áreas verdes, el arbolado urbano y de las redes viarias pensadas originalmente como corredores verdes se encuentran en un proceso de deterioro y falta de mantenimiento hace décadas. Respecto de las vías, su falta de actualización en cuanto al diseño las torna inadecuadas para los nuevos criterios de multimedialidad. Además, debido a las intervenciones que sufrieron en favor del transporte automotor, han perdido en muchos casos su carácter de corredores urbanos y de reservas de la biodiversidad existente en los inicios de la ciudad por la falta de una gestión adecuada que contemple los criterios propuestos en París 2015 y los ODS (Objetivos de Desarrollo Sostenible). Particularmente para el caso de estudio, luego del relevamiento realizado se pudo constatar que existen $8.850 .960 \mathrm{~m}^{2}$ de espacios verdes en el partido, para un total estimado de población de 731.783 personas. Por lo tanto, se constata que si bien la ciudad de La Plata cuenta con más de $10 \mathrm{~m}^{2}$ por habitante (en promedio cuenta con 12,1 $\mathrm{m}^{2} / \mathrm{hab}$.), la accesibilidad a esos espacios no es la misma para todos los habitantes. Esto se debe a la existencia de importantes zonas urbanas críticas en la periferia que no cuentan con espacios de este tipo accesibles para sus habitantes en una caminata de quince minutos. Este requisito solo se cumple en el casco fundacional y en la zona noroeste de la región; el resto de la periferia está desprovista de acceso a espacios verdes urbanos (BIRCHE, 2020; JensEn, 2018). Según el trabajo de Birche (2020), de 121 espacios verdes relevados durante el año 2019, solo 28 tienen calidad considerada como "buena". Estos espacios se ubican en su mayoría dentro del casco fundacional, mientras que los espacios verdes de calidad regular 
Figura 3. Calidad de los espacios verdes para la ciudad de La Plata Fuente: BIRCHE, 2020

\section{Calidad de los espacios verdes para la ciudad de La Plata}

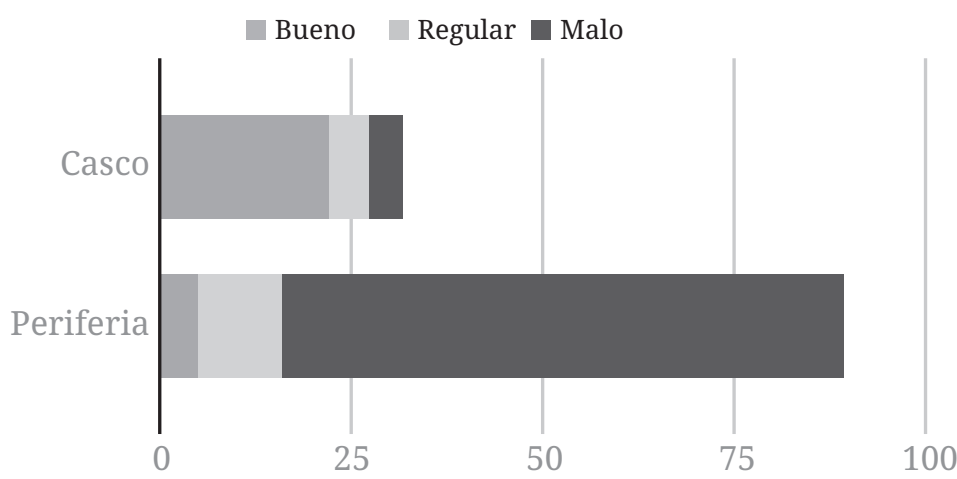

son 21 y los 72 restantes son de calidad mala. Como muestra la figura 3, la mayor parte de los espacios verdes de calidad “mala” se encuentran en el área periférica.

5. Desde la perspectiva socio-ambiental no existen propuestas que impidan determinadas formas de ocupación formal e informal: barrios cerrados en áreas de alta vulnerabilidad ecológica, urbanizaciones informales en áreas inundables sin mínimas condiciones de habitabilidad y la densificación descontrolada en los centros urbanos. Por su parte, las urbanizaciones cerradas muchas veces producen la transformación de las características morfológicas naturales del territorio debido a los requerimientos para su instalación, y provocan la impermeabilización de superficies que impacta sobre el sistema hídrico y de escurrimiento natural.

6. El requerimiento de un organismo intermunicipal para encarar los problemas urbanos y regionales, que ha sido propuesto reiteradamente desde 1960, no ha podido concretarse. Hoy en día la coordinación y la cooperación se constituyen en una necesidad fundamental para enfrentar las intervenciones que no reconocen límites jurisdiccionales en el marco de la reestructuración del espacio globalizado. A continuación, a modo de síntesis del apartado, se presenta el mapa de diagnóstico con aquellas debilidades y fortalezas presentadas en el contexto de la revisión histórica y el análisis de la Región del Gran La Plata. 
Hacia nuevas formas de pensar el territorio:

una propuesta para el desarrollo de la Región del Gran La Plata, Buenos Aires, Argentina

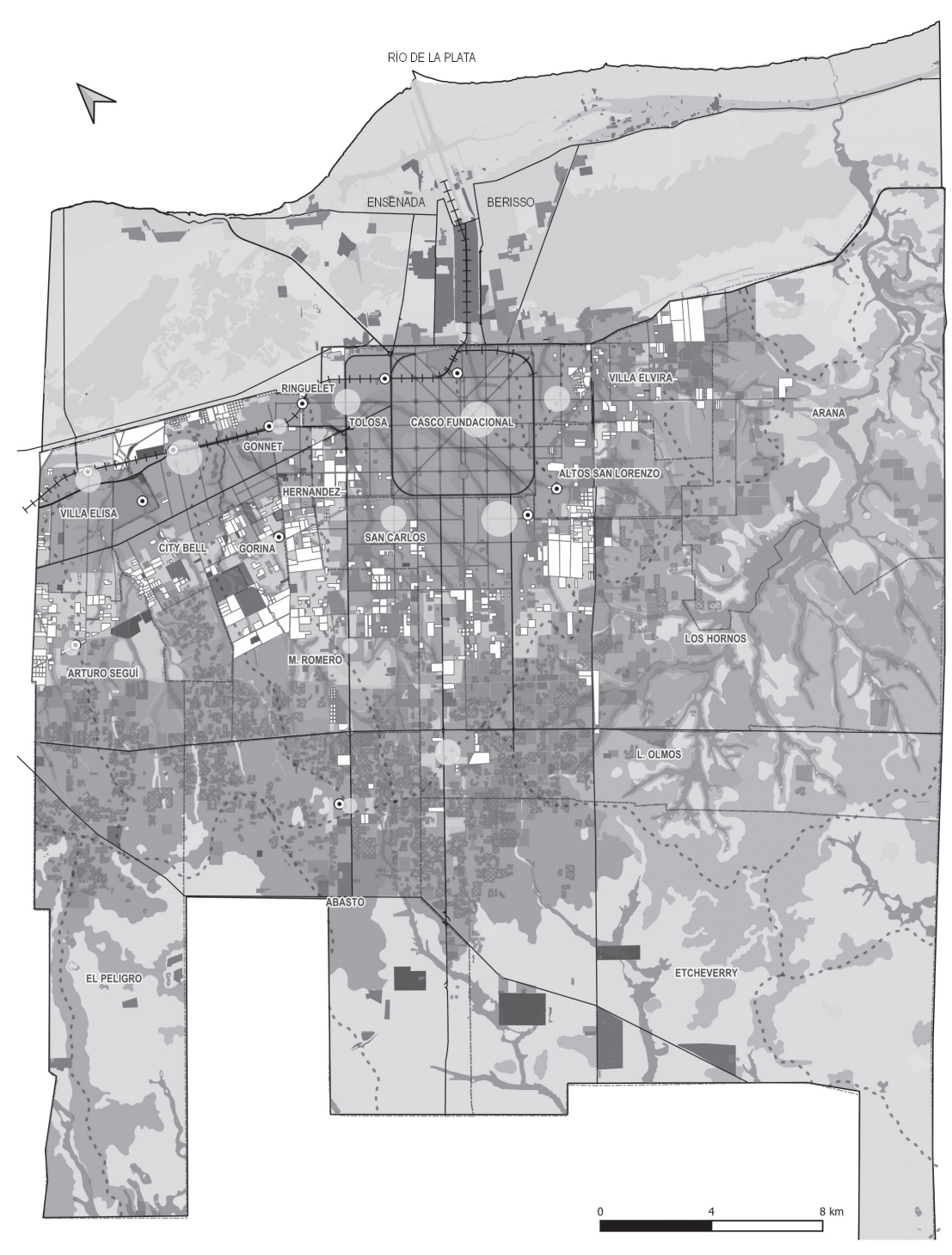

Figura 4. Mapa diagnóstico fortalezas y debilidades Gran La Plata. Fuente: elaboración propia, 2020

\section{REFERENCIAS \\ i.j Delegaciones \\ Red vial \\ $\mapsto+$ Ferrocarriles activos \\ - Estaciones de Ferrocarril \\ Centralidades \\ Expansión urbana \\ Urbanzaciones cerradas \\ Urbanizaciones no registradas \\ Urbanzaciones informales \\ $\square$ Tierras vacantes urbanzables \\ Industrias \\ $18 \% 8$ Invernaderos \\ Cavas \\ Decapitados}

\section{Geomorfologia}

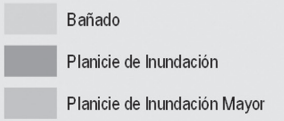

-.. Cuencas Hidrográficas

Clasificación de suelos

I
II
III
IV
IV- VII
VI
VII
VIII




\section{Criterios hacia la sustentabilidad}

Sobre la base del análisis diagnóstico de las problemáticas urbano-territoriales más relevantes en la región, se elaboró una serie de criterios que contemplan también los principios planteados para el diseño fundacional de la ciudad. En la figura 4 se exponen algunas de estas propuestas agrupadas según criterios incluidos en la agenda de quienes plantean un "urbanismo sustentable" o ecológico:

Criterio científico-tecnológico: está orientado a consolidar la infraestructura y los recursos científico-tecnológicos a través de la organización e integración de las distintas áreas del conocimiento y las industrias, y se conforma así en una de las variables principales de la economía en la sociedad del conocimiento. Algunas de sus acciones estarán orientadas a la disposición de nuevas urbanizaciones de "interés social". Además, una propuesta de un polo-tecnológico realizado por la Universidad Nacional de La Plata y aprobado por la provincia de Buenos Aires, que será localizada en uno de los espacios abandonados por una de las estaciones ferroviarias y concebidas según criterios adecuados al medio socio-ambiental. Este espacio incluirá viviendas para el personal del complejo y diversas actividades para el mejoramiento de los espacios públicos, calidad cultural y educativa del entorno barrial. La propuesta se completa con la extensión del tren universitario hasta el predio recuperando una de las vías originales.

Criterio normativo/legal: propone la formulación de normativas y legislación conjunta con la participación de las distintas organizaciones sociales, universitarias y profesionales que tiendan, por una parte, a impedir el crecimiento expansivo y descontrolado de las periferias según lo planteado en el plan Urbis 1961. También se plantean acciones para resguardar y mantener los suelos de alto valor, con producción orgánica reduciendo la utilización de fertilizantes químicos, disminuyendo, en lo posible, el uso de invernaderos, y dedicarlos a la organización de cooperativas de trabajadores, para vincular producción-distribución y comercialización con la inclusión de mercados comunitarios que, localizados en distintos barrios, disminuyan los precios de los productos e incrementen los ingresos de los productores.

Criterio sobre espacios verdes: una red de infraestructura verde, basándose en las ideas planteadas en la fundación de la ciudad y que actualmente se incluyen en el compromiso de 
cambio climático realizado en la COP 21 (Conferencia de las Naciones Unidas sobre el Cambio Climático) en París en 2015, a partir del cual Barcelona se compromete a incrementar $1 \mathrm{~m}^{2}$ por habitante para 2030. En este punto, el concepto de infraestructura verde se constituye en un factor fundamental para mitigar los efectos del cambio climático y aumentar la resiliencia de las ciudades. De esta forma, se proponen para la Región del Gran La Plata:

i) espacios verdes ubicados en tierras vacantes que se encuentren en las zonas críticas sin cobertura de espacios verdes (es decir, aquellas zonas que no puedan acceder caminando en 15 minutos a espacios verdes);

ii) corredores verdes desde la costa del río de La Plata que unan los distintos espacios públicos: áreas vulnerables, urbanizaciones informales y tierras vacantes. Estas deberán posibilitar la diversidad de modos de movilidad y, a su vez, recuperar, en parte, la memoria de la infraestructura viaria arbolada de la ciudad retomando la propuesta realizada en el Plan URBIS;

iii) para las tierras vacantes localizadas en zonas de vulnerabilidad ecológica, se presentan diversas propuestas de acuerdo con las características biofísicas, estructurales y estéticas. Estas propuestas además contemplan la localización de estas tierras y la accesibilidad. Se construyen tres tipos de categorías de espacios verdes: parque urbano, que funciona como un espacio recreativo; parque fluvial, que funciona como un reservorio, y parque de transición, donde se proponen espacios de huerta urbana;

iv) centros recreativos en las tierras pertenecientes a las estaciones ferroviarias en desuso, que integren el patrimonio ferroviario, con espacios públicos y culturales de calidad que incluyan actividades de investigación, capacitación y huertas urbanas, considerando las características de cada uno de sus entornos;

v) espacios verdes refuncionalizados, para el caso de lugares como el exzoológico o el aeródromo, donde se puedan incorporar espacios verdes productivos, viveros municipales y mejorar la calidad ambiental de sus entornos;

vi) espacios de reserva ecológica, como las islas Paulino y Santiago o la reserva Punta Lara.

Criterio sobre nuevas urbanizaciones: las nuevas urbanizaciones estarán ubicadas preferentemente sobre suelos que no sean de alto valor productivo.

En cuanto a la población de menores recursos, se propone, por una parte, reorganizar urbanísticamente aquellas que se encuentren sobre áreas no vulnerables ecológicamente; también se propone despejar zonas que, estando en los márgenes de los arroyos, 


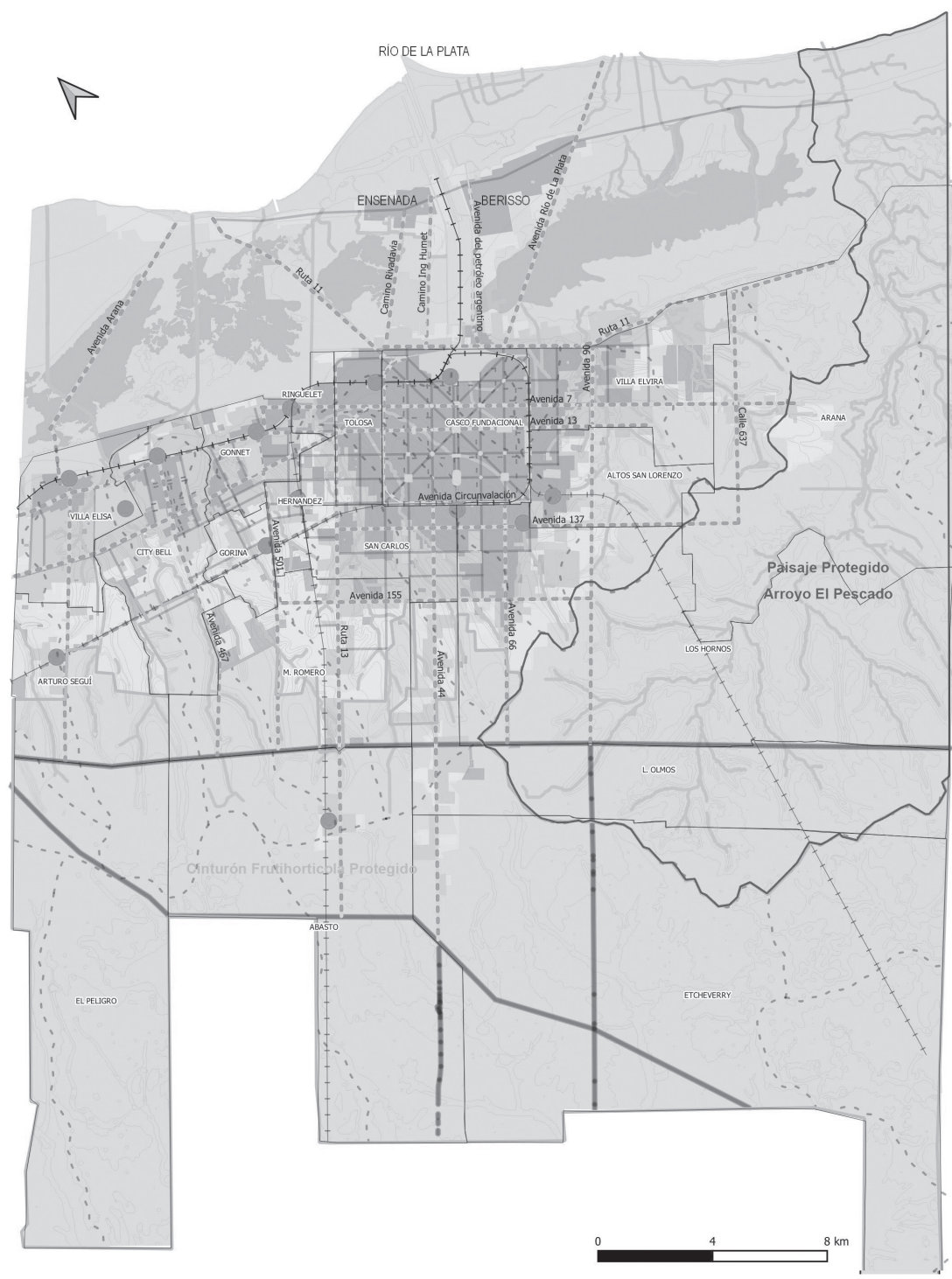

Figura 5. Mapa esquema síntesis propuestas para el Gran La Plata. Fuente: elaboración propia, 2020

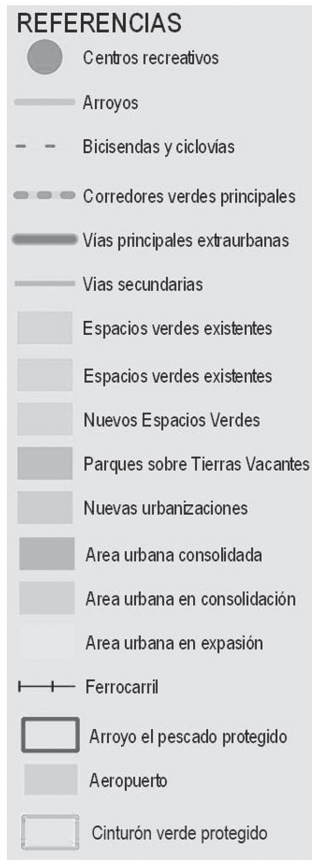


deben adaptarse arquitectónicamente o relocalizarse. Por otra parte, se sugiere que la municipalidad lidere un proceso de desarrollo en aquellas tierras vacantes que cuenten con superficies adecuadas para urbanizaciones de densidad media con viviendas pensadas desde la perspectiva bioclimática. Estas deben incluir tecnologías adecuadas a la problemática ambiental (SchUMACHER, 2001), conservación de la energía, energías renovables vinculadas con diversas formas de trabajo: producción de alimentos y elementos forestales; cuidado de personas mayores, niños y discapacitados y la capacitación en diversas técnicas vinculadas con las tecnologías adecuadas y el mantenimiento de estas.

El futuro crecimiento poblacional del Gran La Plata a partir de estimaciones del censo del INDEC 2010 indica un total de entre 90.000 y 100.000 personas, en una población total de aproximadamente 900.0000 habitantes, entre los cuales 20.000 y 25.000 de ellos corresponden al crecimiento de los sectores que actualmente habitan en las urbanizaciones informales. Parte de ese crecimiento podrá localizarse en las zonas en vías de consolidación a través de diversas estrategias proyectuales, pero también debe pensarse en la posibilidad de crear nuevas urbanizaciones en localidades pequeñas de la región ampliada de La Plata y que cuentan con condiciones ambientales y estructurales adecuadas, las cuales pensadas en red pueden ser vinculadas con los modernos sistemas de transporte (figura 4).

\section{Reflexiones finales}

Gran parte de las propuestas formuladas no son originales; en muchas ciudades del mundo se están desarrollando medidas como las presentadas. Sin embargo, en la Región del Gran La Plata, si bien se proponen algunos proyectos vinculados con el paisaje, la movilidad y el desarrollo urbano, carecen de una concepción que considere las variables de los distintos sistemas que integran el ecosistema urbano. Del análisis desarrollado y con base en las distintas aproximaciones sobre sustentabilidad, es posible afirmar que, si llegaran a concretarse algunas o todas las propuestas, no se logrará alcanzar la denominada "sustentabilidad urbana” mientras aquellas actividades como la petroquímica o la producción hortícola no se transformen integralmente, considerando la "sustentabilidad fuerte". Asimismo, con estas propuestas no se resuelven la inequidad, la exclusión, la marginación, la desigualdad socio-espacial, variables consideradas como parte de la sustentabilidad urbana, si se continúa con el actual modelo ultraliberal. Desde esta perspectiva, un conjunto de economistas europeos, entre ellos Thomas Piketti, sugiere adoptar algunas medidas que actualmente se 
proponen a nivel internacional, como es la renta básica universal, acompañada de políticas culturales y educativas que posibiliten el mejoramiento integral del conjunto social. La realización de estas medidas no significaría disminuir la insustentabilidad global, ya que esta supone adoptar medidas conjuntas de todas las regiones del mundo. Mientras se destruya el Amazonas, se talen bosques nativos, se continúe con las contiendas mundiales y el consumismo, solo será posible lograr disminuir la insustentabilidad local. La propuesta aquí planteada requeriría, en una segunda instancia, la consulta a todos los actores involucrados en la región para posibilitar, localmente, un mejoramiento integral de las condiciones socio-económicas de la población. Esto solo será posible si se realiza en el contexto de una política general de vivir con lo nuestro, como lo proponía Aldo Ferrer, retomando los principios establecidos en el Informe Brundtland (1987) "de satisfacer las necesidades actuales sin comprometer la capacidad de las generaciones futuras para satisfacer las suyas” (ONU, 1987, p. 67).

\section{Referencias bibliográficas}

Birche, M. (2020). El sistema de espacios públicos como factor estructurador de la calidad del paisaje y el ambiente urbano [Tesis de Doctorado]. Facultad de Arquitectura y Urbanismo UNLP). Repositorio SEDICI http://sedici.unlp.edu.ar/ handle/10915/105803.

Cáceres, P. (2003). Análisis cualitativo de contenido: una alternativa. Metodología alternativa. Sico perspectivas, revista de la escuela de psicología facultad de filosofía y educación pontificia universidad católica de Valparaíso (2), 53-82. $\mathrm{http://10.5027/psicoperspectivas-Vol2-Issue1-fulltext-3}$

Gorelik, A. (2004). Miradas sobre Buenos Aires. Historia cultural y crítica urbana. Siglo XXI. Henríquez, C. (2004). Reflexiones sobre la Ciudad Sostenible, Modelos e Indicadores Urbanos. Biblioteca Digital DIBRI -UCSH Universidad Católica Silva Henríquez UCSH -DIBRI.

Jensen, K. (2018). Paisajes vacantes. El paisaje y los espacios verdes en la periferia platense. [Tesis de doctorado]. Facultad de Arquitectura y Urbanismo, UNLP. Repositorio SEDICI http://sedici.unlp.edu.ar/handle/10915/72319

Lefebvre, H. (1972). La revolución urbana. Traducción de Mario Nolla. Alianza Editorial.

Naredo, José Manuel (1996). Sistema de cuentas de los recursos naturales y el medio ambiente, ponencia presentada en III Congreso Nacional del Medio Ambiente, 25-29 de noviembre, Madrid. 
Naredo, J. (2004). Sobre el origen, el uso y el contenido del término sostenible. Cuadernos de Investigación Urbanística, 0(41). http://polired.upm.es/index.php/ciur/ article/view/1032

Naredo, J. (2013). Ideología político económica dominante y claves para un nuevo paradigma. Revista de economía crítica, (16), 108-143.

ONU (1987). Nuestro futuro común. Alianza.

Piketty, T. (2015). El capital en el siglo XX. Revista Latinoamericana de Población, 9 (17), 169-172.

Ravella, O. \& Giacobbe, N. (2001). La planificación urbana regional. UNLP.

Rocha, D. (1881). Discurso pronunciado el 1 de mayo de 1881, frente a la Legislatura al asumir sus funciones de Gobernador de la Provincia de Buenos Aires.

Rodríguez Tarducci, R. (2020). Informalidad urbana en el partido de La Plata: Análisis del proceso de ocupación y apropiación territorial, 1989-actualidad. [Tesis de doctorado]. Universidad Nacional de La Plata UNLP. Repositorio SEDICI http://sedici.unlp.edu.ar/handle/10915/95859

Sachs, I. (1981). Ecodesarrollo. Agricultura y sociedad, (18), 9-32.

Schumacher, E. F. (2001). Lo pequeño es hermoso. AKAL

Vallejo, G. (2005). El trazado ideal y los condicionantes naturales en el desarrollo urbano de La Plata. Estudios del Hábitat, Vol. II, N. ${ }^{\circ}$, pp. 61-70. 
